\title{
POSITIVE SOLUTIONS IN THE SENSE OF DISTRIBUTIONS OF SINGULAR BOUNDARY VALUE PROBLEMS
}

\author{
RAVI P. AGARWAL, KANISHKA PERERA, AND DONAL O'REGAN \\ (Communicated by Carmen C. Chicone)
}

\begin{abstract}
We obtain positive solutions in the sense of distributions of singular boundary value problems using perturbation and variational methods.
\end{abstract}

\section{INTRODUCTION}

In recent years fixed point theory and other methods have been used to obtain positive solutions of singular boundary value problems such as

$$
\left\{\begin{array}{l}
-u^{\prime \prime}=u^{-q}+g(x, u), \quad 0<x<1, \\
u(0)=u(1)=0
\end{array}\right.
$$

with certain restrictions on $q>0$ and various assumptions on the regular term $g$; see, e.g., Agarwal and O'Regan [1] for an extensive bibliography. In this paper we use perturbation and variational methods to obtain new existence and multiplicity results for a broad class of singular problems that includes (1.1) with no restrictions on $q$. Our techniques are applicable to other types of singular problems as well.

We consider the problem

$$
\begin{cases}-u^{\prime \prime}=f(x, u)+g_{0}(x, u)+\mu g_{1}(x, u), & 0<x<1, \\ u(x)>0, & 0<x<1, \\ u(0)=u(1)=0 & \end{cases}
$$

where $f, g_{0}, g_{1} \in C((0,1) \times(0, \infty), \mathbb{R})$ satisfy

$\left(A_{1}\right) \exists t_{0}>0$ and a nontrivial bounded function $f_{0} \geq 0$ in $C(0,1)$ such that $f(x, t) \geq f_{0}(x), g_{0}(x, t), g_{1}(x, t) \geq 0$ for $t \leq t_{0}$,

$\left(A_{2}\right) \sup _{(x, s) \in(0,1) \times[t, \infty)}|f(x, s)|, \sup _{(x, s) \in(0,1) \times(0, t]}\left|g_{1}(x, s)\right|<\infty$ for each $t$,

$\left(A_{3}\right)\left|g_{0}(x, t)\right| \leq \lambda t+a_{0}$ for some constants $\lambda<\lambda_{1}$, the first Dirichlet eigenvalue of $-\frac{d^{2}}{d x^{2}}$ on $(0,1)$, and $a_{0} \geq 0$

Received by the editors November 14, 2005 and, in revised form, January 22, 2007.

2000 Mathematics Subject Classification. Primary 34B16; Secondary 47J30.

Key words and phrases. Singular boundary value problems, positive solutions in the sense of distributions, perturbation and variational methods. 
and $\mu \geq 0$ is a small parameter.

Recall that a weak solution of (1.2) is a positive function $u$ in the Sobolev space $H_{0}^{1}(0,1)$ satisfying

$$
\int_{0}^{1}\left[u^{\prime}(x) \varphi^{\prime}(x)-\left(f(x, u)+g_{0}(x, u)+\mu g_{1}(x, u)\right) \varphi(x)\right] d x=0
$$

for all $\varphi \in H_{0}^{1}(0,1)$. We seek solutions in the sense of distributions: a solution of (1.2) is a function $u \in H_{\mathrm{loc}}^{1}(0,1) \cap C_{0}[0,1]$, where $C_{0}[0,1]$ is the space of continuous functions on $[0,1]$ that vanish on the boundary, that is positive in $(0,1)$ and satisfies (1.3) for all test functions $\varphi \in C_{0}^{\infty}(0,1)$. Then $u$ is in the Hölder space $C_{\text {loc }}^{1, \alpha}(0,1)$ for any $\alpha<1$ by standard regularity arguments.

Theorem 1.1. If $\left(A_{1}\right)-\left(A_{3}\right)$ hold, then $\exists \mu_{0}>0$ such that (1.2) has a solution $u_{1}$ for each $\mu \in\left[0, \mu_{0}\right)$.

Theorem 1.2. If $f(x, t)$ is bounded on compact $t$ intervals and nonincreasing in $t$, then (1.2) with $g_{0}=g_{1}=0$ has at most one solution.

Now we assume

$\left(A_{4}\right) f(x, t)$ is nonincreasing and convex in $t$ for $t \leq t_{0}$,

$\left(A_{5}\right)\left|g_{1}(x, t)\right| \leq a_{1} t^{p-1}+a_{2}$ for some $p>2$ and $a_{1}, a_{2} \geq 0$,

$\left(A_{6}\right) \exists t_{1}>0$ such that $0<G_{1}(x, t):=\int_{0}^{t} g_{1}(x, s) d s \leq \frac{1}{p} \operatorname{tg} g_{1}(x, t)$ for $t \geq t_{1}$.

Theorem 1.3. If $\left(A_{1}\right)-\left(A_{6}\right)$ hold, then $\exists \mu_{0}>0$ such that (1.2) has two solutions $u_{1} \leq u_{2}$ with $u_{2}-u_{1} \in H_{0}^{1}(0,1)$ for each $\mu \in\left(0, \mu_{0}\right)$.

Example 1.4. The problem

$$
\begin{cases}-u^{\prime \prime}=e^{1 / u}+\lambda u+\mu u^{p-1}, & 0<x<1, \\ u(x)>0, & 0<x<1, \\ u(0)=u(1)=0, & \end{cases}
$$

where $p>2$, has

(i) a unique solution if $\lambda=\mu=0$,

(ii) at least one solution if $0<\lambda<\lambda_{1}$ and $\mu=0$,

(iii) two ordered solutions if $0 \leq \lambda<\lambda_{1}$ and $\mu>0$ is sufficiently small.

We will make use of the following variant of the mountain pass lemma due to Cerami [2] in getting the second solution in Theorem 1.3.

Proposition 1.5. If $\Phi$ is a $C^{1}$ functional defined on a Banach space $H$, and $\exists v_{0}, v_{1} \in H$ such that

$$
c:=\inf _{\gamma \in \Gamma} \max _{v \in \gamma([0,1])} \Phi(v)>\Phi\left(v_{0}\right), \Phi\left(v_{1}\right)
$$

where

$$
\Gamma:=\left\{\gamma \in C([0,1], H): \gamma(0)=v_{0}, \gamma(1)=v_{1}\right\}
$$

is the class of paths in $H$ joining $v_{0}$ and $v_{1}$, then there is a sequence $\left(v_{j}\right) \subset H$ such that

$$
\left|\Phi\left(v_{j}\right)-c\right| \rightarrow 0, \quad\left(1+\left\|v_{j}\right\|\right)\left\|\Phi^{\prime}\left(v_{j}\right)\right\| \rightarrow 0
$$




\section{Preliminaries}

Consider

$$
\begin{cases}-u^{\prime \prime}=h(x, u), & 0<x<1, \\ u(x)>0, & 0<x<1, \\ u(0)=u(1)=0 & \end{cases}
$$

where $h=f+g$ and $f, g \in C((0,1) \times(0, \infty), \mathbb{R})$ satisfy

$$
\sup _{(x, s) \in(0,1) \times\left[t_{1}, t_{2}\right]}|f(x, s)|, \sup _{(x, s) \in(0,1) \times\left(0, t_{2}\right]}|g(x, s)|<\infty \quad \forall 0<t_{1} \leq t_{2}<\infty .
$$

We approximate (2.1) with the sequence of regular problems

$$
\left\{\begin{array}{l}
-u^{\prime \prime}=f_{j}(x, u)+g(x, u), \quad 0<x<1, \\
u(0)=u(1)=0
\end{array}\right.
$$

where $f_{j}(x, t)=f\left(x, \max \left\{t, \varepsilon_{j}\right\}\right)$ and $\left(\varepsilon_{j}\right)$ is a sequence of positive numbers decreasing to zero.

Proposition 2.1. If $\left(u_{j}\right) \subset H_{0}^{1}(0,1)$ is a sequence of weak solutions of (2.3) such that

$$
\begin{gathered}
\varepsilon_{\delta}:=\inf _{j} \min _{[\delta, 1-\delta]} u_{j}>0 \quad \forall 0<\delta \leq 1 / 2, \\
M:=\sup _{j} \max _{[0,1]} u_{j}<\infty,
\end{gathered}
$$

then a subsequence converges pointwise to a solution $u_{1}$ of (2.1).

Proof. Take a sequence $\left(\delta_{k}\right)$ of positive numbers decreasing to zero. For all $j$ so large that $\varepsilon_{j}<\varepsilon_{\delta_{1}}$, taking $\left(u_{j}-\varepsilon_{\delta_{1}}\right)^{+}$as the test function in (2.3) gives

$$
\int_{\left\{u_{j}(x)>\varepsilon_{\delta_{1}}\right\}} u_{j}^{\prime}(x)^{2} d x=\int_{\left\{u_{j}(x)>\varepsilon_{\delta_{1}}\right\}} h\left(x, u_{j}\right)\left(u_{j}(x)-\varepsilon_{\delta_{1}}\right) d x .
$$

Since $u_{j} \geq \varepsilon_{\delta_{1}}$ on $\left[\delta_{1}, 1-\delta_{1}\right]$ by (2.4) and the right side is bounded by (2.2) and (2.5), $\left(u_{j}\right)$ is bounded in $H^{1}\left(\delta_{1}, 1-\delta_{1}\right)$ and hence a subsequence $\left(u_{1, j}\right)$ converges to some $u^{1}$ weakly in $H^{1}\left(\delta_{1}, 1-\delta_{1}\right)$ and strongly in $C\left[\delta_{1}, 1-\delta_{1}\right]$. Repeating with further and further subsequences, for each $k$ we get a subsequence $\left(u_{k j}\right)$ that converges to some $u^{k}$ weakly in $H^{1}\left(\delta_{k}, 1-\delta_{k}\right)$ and strongly in $C\left[\delta_{k}, 1-\delta_{k}\right]$ with $\left(u_{k+1, j}\right) \subset\left(u_{k j}\right)$. Then $\left.u^{k+1}\right|_{\left[\delta_{k}, 1-\delta_{k}\right]}=u^{k}$, so

$$
u_{1}:= \begin{cases}u^{1} & \text { on }\left[\delta_{1}, 1-\delta_{1}\right], \\ u^{k+1} & \text { on }\left[\delta_{k+1}, 1-\delta_{k+1}\right] \backslash\left[\delta_{k}, 1-\delta_{k}\right] \text { for each } k\end{cases}
$$

is a well-defined positive function in $H_{\mathrm{loc}}^{1}(0,1) \cap C(0,1)$, to which the diagonal subsequence $\left(u_{k k}\right)$ converges pointwise.

To see that $u_{1} \in C_{0}[0,1]$, let $0<\varepsilon<M, M_{\varepsilon}=\sup h((0,1) \times[\varepsilon, M])>0$, and $\varphi_{\varepsilon}>0$ be the solution of

$$
\left\{\begin{aligned}
-\varphi_{\varepsilon}^{\prime \prime} & =M_{\varepsilon}, \quad 0<x<1, \\
\varphi_{\varepsilon}(0) & =\varphi_{\varepsilon}(1)=0
\end{aligned}\right.
$$


For all $k$ so large that $\varepsilon_{k k}<\varepsilon$, taking $\varphi=\left(u_{k k}-\varepsilon-\varphi_{\varepsilon}\right)^{+}$as the test function in

$$
-u_{k k}^{\prime \prime}=f_{k k}\left(x, u_{k k}\right)+g\left(x, u_{k k}\right)
$$

gives

$$
\begin{aligned}
\int_{0}^{1} u_{k k}^{\prime}(x) \varphi^{\prime}(x) d x & =\int_{0}^{1} h\left(x, u_{k k}\right) \varphi(x) d x \\
& \leq \int_{0}^{1} M_{\varepsilon} \varphi(x) d x=\int_{0}^{1} \varphi_{\varepsilon}^{\prime}(x) \varphi^{\prime}(x) d x
\end{aligned}
$$

by $(2.8)$, so

$$
\int_{\left\{u_{k k}(x)>\varepsilon+\varphi_{\varepsilon}(x)\right\}}\left(u_{k k}^{\prime}(x)-\varphi_{\varepsilon}^{\prime}(x)\right)^{2} d x \leq 0
$$

and hence $u_{k k} \leq \varepsilon+\varphi_{\varepsilon}$. Thus $0<u_{1} \leq \varepsilon+\varphi_{\varepsilon}$, which implies that $u_{1}(x) \rightarrow 0$ as $x \rightarrow 0,1$ since $\varphi_{\varepsilon}(x) \rightarrow 0$ as $x \rightarrow 0,1$ and $\varepsilon$ is arbitrary.

For any $\varphi \in C_{0}^{\infty}(0,1)$,

$$
\int_{\delta_{k}}^{1-\delta_{k}}\left[u_{k j}^{\prime}(x) \varphi^{\prime}(x)-h\left(x, u_{k j}\right) \varphi(x)\right] d x=0
$$

for a fixed $k$ so large that $\left[\delta_{k}, 1-\delta_{k}\right] \supset \operatorname{supp} \varphi$ and all $j$ so large that $\varepsilon_{k j}<\varepsilon_{\delta_{k}}$, and passing to the limit gives

$$
\int_{\delta_{k}}^{1-\delta_{k}}\left[\left(u^{k}\right)^{\prime}(x) \varphi^{\prime}(x)-h\left(x, u^{k}\right) \varphi(x)\right] d x=0,
$$

or

$$
\int_{0}^{1}\left[u_{1}^{\prime}(x) \varphi^{\prime}(x)-h\left(x, u_{1}\right) \varphi(x)\right] d x=0
$$

since $u^{k}=\left.u_{1}\right|_{\left[\delta_{k}, 1-\delta_{k}\right]}$ and $\varphi=0$ outside $\left[\delta_{k}, 1-\delta_{k}\right]$.

Proposition 2.2. If (2.3) has a sequence of weak sub-and supersolution pairs $\underline{u}_{j} \leq \bar{u}_{j}$ in $H_{0}^{1}(0,1)$ such that

$$
\inf _{j} \min _{[\delta, 1-\delta]} \underline{u}_{j}>0 \quad \forall 0<\delta \leq 1 / 2, \quad \sup _{j} \max _{[0,1]} \bar{u}_{j}<\infty,
$$

then (2.1) has a solution $u_{1}$.

Proof. By a standard argument (2.3) has a weak solution $u_{j} \in H_{0}^{1}(0,1)$ in the order interval $\left[\underline{u}_{j}, \bar{u}_{j}\right]$, and the conclusion follows from Proposition 2.1.

Now we assume that $f(x, t)$ is nonincreasing in $t$. Given a solution $u_{1}$ of $(2.1)$ we seek a second solution of the form $u_{2}=u_{1}+v$ where $v \geq 0$ is then a solution of

$$
\left\{\begin{array}{l}
-v^{\prime \prime}=h\left(x, u_{1}+v\right)-h\left(x, u_{1}\right), \quad 0<x<1, \\
v(0)=v(1)=0
\end{array}\right.
$$

Let $\left(\delta_{j}\right)$ be a sequence of positive numbers decreasing to zero and consider the approximating sequence of regular problems

$$
\left\{\begin{array}{l}
-v^{\prime \prime}=h\left(x, u_{1}+v^{+}\right)-h\left(x, u_{1}\right), \quad \delta_{j}<x<1-\delta_{j}, \\
v\left(\delta_{j}\right)=v\left(1-\delta_{j}\right)=0 .
\end{array}\right.
$$


Solutions of (2.17) are nonnegative by the maximum principle and coincide with the critical points of the $C^{1}$ functional

$$
\Phi_{j}(v)=\int_{\delta_{j}}^{1-\delta_{j}}\left[\frac{1}{2} v^{\prime}(x)^{2}-\int_{0}^{v(x)^{+}}\left(h\left(x, u_{1}+s\right)-h\left(x, u_{1}\right)\right) d s\right] d x
$$

defined on $H_{0}^{1}\left(\delta_{j}, 1-\delta_{j}\right)$, which we view as a subspace of $H_{0}^{1}(0,1)$ by setting each $v=0$ outside $\left(\delta_{j}, 1-\delta_{j}\right)$.

Proposition 2.3. If $\left(v_{j}\right), v_{j} \in H_{0}^{1}\left(\delta_{j}, 1-\delta_{j}\right)$ is a bounded sequence in $H_{0}^{1}(0,1)$ such that

$$
\inf _{j} \Phi_{j}\left(v_{j}\right)>0, \quad\left\|\Phi_{j}^{\prime}\left(v_{j}\right)\right\| \rightarrow 0
$$

$\left(\|\cdot\|\right.$ is the norm in $\left.H^{-1}\left(\delta_{j}, 1-\delta_{j}\right)\right)$, then a subsequence converges pointwise to a nontrivial function $v \geq 0$ in $H_{0}^{1}(0,1)$ such that $u_{2}=u_{1}+v$ is a solution of (2.1).

Proof. Since $\left(v_{j}\right)$ is bounded in $H_{0}^{1}(0,1)$, a subsequence converges to some $v$ weakly in $H_{0}^{1}(0,1)$ and strongly in $C_{0}[0,1]$. Since

$$
\left\|v_{j}^{-}\right\|^{2}=\Phi_{j}^{\prime}\left(v_{j}\right) v_{j}^{-} \rightarrow 0
$$

by (2.19), $v \geq 0$ and hence $u_{2} \geq u_{1}>0$ in $(0,1)$.

For any $\varphi \in C_{0}^{\infty}(0,1)$ and all $j$ so large that $\left[\delta_{j}, 1-\delta_{j}\right] \supset \operatorname{supp} \varphi$,

$$
\Phi_{j}^{\prime}\left(v_{j}\right) \varphi=\int_{0}^{1}\left[v_{j}^{\prime}(x) \varphi^{\prime}(x)-\left(h\left(x, u_{1}+v_{j}^{+}\right)-h\left(x, u_{1}\right)\right) \varphi(x)\right] d x,
$$

and passing to the limit and adding

$$
\int_{0}^{1}\left[u_{1}^{\prime}(x) \varphi^{\prime}(x)-h\left(x, u_{1}\right) \varphi(x)\right] d x=0
$$

gives

$$
\int_{0}^{1}\left[u_{2}^{\prime}(x) \varphi^{\prime}(x)-h\left(x, u_{2}\right) \varphi(x)\right] d x=0 .
$$

Since $f(x, t)$ is nonincreasing in $t$,

$$
\begin{aligned}
& \int_{0}^{1}\left[\int_{0}^{v_{j}(x)^{+}}\left(f\left(x, u_{1}+s\right)-f\left(x, u_{1}\right)\right) d s\right] d x \\
\geq & \int_{0}^{1}\left(f\left(x, u_{1}+v_{j}^{+}\right)-f\left(x, u_{1}\right)\right) v_{j}(x)^{+} d x \\
= & \left\|v_{j}^{+}\right\|^{2}-\int_{0}^{1}\left(g\left(x, u_{1}+v_{j}^{+}\right)-g\left(x, u_{1}\right)\right) v_{j}(x)^{+} d x-\Phi_{j}^{\prime}\left(v_{j}\right) v_{j}^{+}
\end{aligned}
$$

and hence

$$
\begin{aligned}
\Phi_{j}\left(v_{j}\right) \leq & \frac{1}{2}\left\|v_{j}^{-}\right\|^{2}-\int_{0}^{1}\left[\int_{0}^{v_{j}(x)^{+}}\left(g\left(x, u_{1}+s\right)-g\left(x, u_{1}\right)\right) d s\right. \\
& \left.-\left(g\left(x, u_{1}+v_{j}^{+}\right)-g\left(x, u_{1}\right)\right) v_{j}(x)^{+}\right] d x+\Phi_{j}^{\prime}\left(v_{j}\right) v_{j}^{+} .
\end{aligned}
$$

The right side goes to zero by (2.2) and (2.20) if $v=0$, contrary to (2.19). 


\section{Proofs}

Proof of Theorem 1.1. We apply Proposition 2.2 with $g=g_{0}+\mu g_{1}$. Let $t_{0}$ and $f_{0}$ be as in $\left(A_{1}\right)$ and let $0<\varepsilon \leq 1$ be so small that the solution $\underline{u}>0$ in $H_{0}^{1}(0,1)$ of

$$
\left\{\begin{array}{l}
-u^{\prime \prime}=\varepsilon f_{0}(x), \quad 0<x<1 \\
u(0)=u(1)=0
\end{array}\right.
$$

is $\leq t_{0}$. Then

$$
-\underline{u}^{\prime \prime} \leq f_{0}(x) \leq f_{j}(x, \underline{u})+g(x, \underline{u})
$$

for all $j$ so large that $\varepsilon_{j}<t_{0}$ and $\mu \geq 0$.

By $\left(A_{2}\right)$ and $\left(A_{3}\right)$,

$$
\left\{\begin{array}{l}
-u^{\prime \prime}=f_{j}(x, u)+g_{0}(x, u)+1, \quad 0<x<1, \\
u(0)=u(1)=0
\end{array}\right.
$$

has a solution $\bar{u}_{j} \in H_{0}^{1}(0,1)$. By $\left(A_{1}\right)$ and the maximum principle, $\bar{u}_{j} \geq \underline{u}$. Taking $\left(\bar{u}_{j}-t_{0}\right)^{+}$as the test function in (3.3) gives

$$
\left\|\left(\bar{u}_{j}-t_{0}\right)^{+}\right\|^{2} \leq \int_{\left\{\bar{u}_{j}(x)>t_{0}\right\}}\left(\lambda \bar{u}_{j}(x)+a_{3}\right)\left(\bar{u}_{j}(x)-t_{0}\right) d x
$$

for some $a_{3} \geq 0$, so $\left(\left(\bar{u}_{j}-t_{0}\right)^{+}\right)$is bounded in $H_{0}^{1}(0,1)$ and hence $\left(\bar{u}_{j}\right)$ is bounded in $C_{0}[0,1]$. Then

$$
-\bar{u}_{j}^{\prime \prime} \geq f_{j}\left(x, \bar{u}_{j}\right)+g_{0}\left(x, \bar{u}_{j}\right)+\mu g_{1}\left(x, \bar{u}_{j}\right)
$$

for all sufficiently small $\mu \geq 0$.

Proof of Theorem 1.2. Suppose $u_{1}$ and $u_{2}$ are both solutions. For any $\varepsilon>0$, taking $u=u_{1}, u_{2}$ with $\varphi=\left(u_{1}-u_{2}-\varepsilon\right)^{+}$in (1.3) and subtracting gives

$$
\begin{aligned}
& \int_{\left\{u_{1}(x)>u_{2}(x)+\varepsilon\right\}}\left(u_{1}^{\prime}(x)-u_{2}^{\prime}(x)\right)^{2} d x \\
= & \int_{\left\{u_{1}(x)>u_{2}(x)+\varepsilon\right\}}\left(f\left(x, u_{1}\right)-f\left(x, u_{2}\right)\right)\left(u_{1}(x)-u_{2}(x)-\varepsilon\right) d x \leq 0
\end{aligned}
$$

since $f(x, t)$ is nonincreasing in $t$, so $u_{1} \leq u_{2}+\varepsilon$. Since $\varepsilon$ is arbitrary, $u_{1} \leq u_{2}$, and the reverse inequality follows similarly.

Proof of Theorem 1.3. We apply Proposition 1.5 to $\Phi_{j}$ and use Proposition 2.3 to get $u_{2}$. By $\left(A_{2}\right)$ and $\left(A_{4}\right)$, we may assume that $f(x, t) \geq 0$ and nonincreasing and convex in $t$ for all $t$ by replacing $f(x, t)$ and $g_{0}(x, t)$ with $f\left(x, t_{0}\right)$ and $g_{0}(x, t)+$ $f(x, t)-f\left(x, t_{0}\right)$ for $t>t_{0}$, respectively.

Since $f$ is nonincreasing in $t$,

$$
\int_{0}^{v(x)^{+}}\left(f\left(x, u_{1}+s\right)-f\left(x, u_{1}\right)\right) d s \leq 0,
$$

and hence

$$
\Phi_{j}(v) \geq \frac{1}{2}\left(1-\frac{\lambda}{\lambda_{1}}\right)\|v\|^{2}-a_{4}\left(1+\mu\|v\|^{p-1}\right)\|v\| \quad \forall v
$$


for some $a_{4} \geq 0$ by $\left(A_{3}\right)$ and $\left(A_{5}\right)$. So $\exists c_{0}, R, \mu_{0}>0$ such that

$$
\inf _{v \in H_{0}^{1}\left(\delta_{j}, 1-\delta_{j}\right),\|v\|=R} \Phi_{j}(v) \geq c_{0} \quad \forall \mu \in\left(0, \mu_{0}\right) .
$$

Since $f(x, t) \geq 0$,

$$
\int_{0}^{1}\left[\int_{0}^{v(x)^{+}}\left(f\left(x, u_{1}+s\right)-f\left(x, u_{1}\right)\right) d s\right] d x \geq-\int_{0}^{1} f\left(x, u_{1}\right) v(x)^{+} d x,
$$

and integrating $\left(A_{6}\right)$ gives

$$
g_{1}(x, t) \geq a_{5} t^{p-1}, \quad t \geq t_{1},
$$

for some $a_{5}>0$, so for each $\mu>0, \exists v_{1}>0$ in $H_{0}^{1}\left(\delta_{1}, 1-\delta_{1}\right),\left\|v_{1}\right\|>R$ such that

$$
\Phi_{j}\left(v_{1}\right)=\Phi_{1}\left(v_{1}\right) \leq 0 \quad \forall j .
$$

Noting that $\Phi_{j}(0)=0$ and setting

$$
c_{j}:=\inf _{\gamma \in \Gamma_{j}} \max _{v \in \gamma([0,1])} \Phi_{j}(v) \geq c_{0}
$$

where

$$
\Gamma_{j}:=\left\{\gamma \in C\left([0,1], H_{0}^{1}\left(\delta_{j}, 1-\delta_{j}\right)\right): \gamma(0)=0, \gamma(1)=v_{1}\right\},
$$

Proposition 1.5 now gives a $v_{j} \in H_{0}^{1}\left(\delta_{j}, 1-\delta_{j}\right)$ such that

$$
\left|\Phi_{j}\left(v_{j}\right)-c_{j}\right| \rightarrow 0, \quad\left(1+\left\|v_{j}\right\|\right)\left\|\Phi_{j}^{\prime}\left(v_{j}\right)\right\| \rightarrow 0 .
$$

Since $H_{0}^{1}\left(\delta_{j}, 1-\delta_{j}\right) \subset H_{0}^{1}\left(\delta_{j+1}, 1-\delta_{j+1}\right), \Gamma_{j} \subset \Gamma_{j+1}$ and hence $c_{j} \geq c_{j+1}$, so

$$
\frac{c_{0}}{2} \leq \Phi_{j}\left(v_{j}\right) \leq 2 c_{1}
$$

for all sufficiently large $j$.

We have

$$
\begin{aligned}
\Phi_{j}\left(v_{j}\right)-\frac{1}{2} \Phi_{j}^{\prime}\left(v_{j}\right) v_{j}^{+}=\frac{1}{2}\left\|v_{j}^{-}\right\|^{2} & +\int_{0}^{1}\left[\frac{1}{2}\left(h\left(x, u_{1}+v_{j}^{+}\right)+h\left(x, u_{1}\right)\right) v_{j}(x)^{+}\right. \\
& \left.-\int_{u_{1}(x)}^{u_{1}(x)+v_{j}(x)^{+}} h(x, s) d s\right] d x
\end{aligned}
$$

where $h=f+g$. Since $f(x, t)$ is convex in $t$,

$$
\frac{1}{2}\left(f\left(x, u_{1}+v_{j}^{+}\right)+f\left(x, u_{1}\right)\right) v_{j}^{+}-\int_{u_{1}}^{u_{1}+v_{j}^{+}} f(x, s) d s \geq 0,
$$

and $\left(A_{3}\right),\left(A_{5}\right),\left(A_{6}\right)$, and (3.11) imply that the integrals of the corresponding expressions for $g_{0}$ and $g_{1}$ are bounded from below by $-a_{6}\left(\left\|v_{j}^{+}\right\|_{L^{2}(0,1)}^{2}+1\right)$ and $a_{7}\left\|v_{j}^{+}\right\|_{L^{p}(0,1)}^{p}-a_{8}$ for some $a_{6}, a_{8} \geq 0, a_{7}>0$, respectively. So $\left(v_{j}^{+}\right)$is bounded in $L^{p}(0,1)$, and hence it follows from

$$
\frac{1}{2}\left\|v_{j}\right\|^{2} \leq \Phi_{j}\left(v_{j}\right)+\int_{0}^{1}\left[\int_{0}^{v_{j}(x)^{+}}\left(g\left(x, u_{1}+s\right)-g\left(x, u_{1}\right)\right) d s\right] d x
$$

that $\left(v_{j}\right)$ is bounded in $H_{0}^{1}(0,1)$. The conclusion follows since (2.19) holds by (3.15) and (3.16). 


\section{REFERENCES}

[1] Ravi P. Agarwal and Donal O'Regan. Singular differential and integral equations with applications. Kluwer Academic Publishers, Dordrecht, 2003. MR2011127 (2004h:34002)

[2] Giovanna Cerami. An existence criterion for the critical points on unbounded manifolds. Istit. Lombardo Accad. Sci. Lett. Rend. A, 112(2):332-336 (1979), 1978. MR581298 (81k:58021)

Department of Mathematical Sciences, Florida Institute of Technology, Melbourne, FLORIDA 32901

E-mail address: agarwal@fit.edu

Department of Mathematical Sciences, Florida Institute of Technology, Melbourne, FLORIDA 32901

E-mail address: kperera@fit.edu

Department of Mathematics, National University of Ireland, Galway, Ireland

E-mail address: donal.oregan@nuigalway.ie 